\title{
Recyclable Soluble Polyisobutylene-Bound Oxidizing Agents
}

\author{
Peerada Samunual, David E. Bergbreiter* \\ Department of Chemistry, Texas A\&M University, PO Box 30012, College Station, TX 77842-3012, USA
}

\section{ARTICLE INFO}

\section{Article history}

Received

Received in revised form

Accepted

Available online

Keywords:

Oxidation

Recycling

Green Chemistry

Polymer Supported Reagent

Polyisobutylene

\section{ABSTRACT}

This paper describes the use of alkene-terminated polyisobutylene (PIB) as a support for oxidizing agents. Two oxidizing agents, an ionically immobilized PIB-bound perruthenate oxidation catalyst and a PIB-bound 2-iodoxybenzoic acid (IBX) oxidizing reagent, were studied. A perruthenate catalyst ionically bound to PIB was prepared by binding the perruthenate anion to PIB that contained either a terminal tetraalkylammonium or an imidazolium salt. The PIB supported ammonium and imidazolium perruthenate were both selectively soluble in heptane and effective in the oxidation of alcohols. While these PIB-bound perruthenate catalysts could be readily separated from products, recycling was less successful. A PIB bound 2iodoxybenzoic (IBX) oxidizing agent was coupled to a PIB support with an ester bond. It was highly soluble in nonpolar and moderately polar organic solvents. By taking advantage of PIB's heptane solubility, the spent IBX reagent could be easily separated from products by a liquid/liquid heptane/acetonitrile biphasic separation. The recovered IBX reagent could then be regenerated and used in multiple cycles for oxidation of both primary and secondary alcohols.

2009 Elsevier Ltd. All rights reserved.

\section{Introduction}

Alkene-terminated polyisobutylene is a commercially available, nontoxic, and inexpensive nonpolar polymer that is soluble in solvents such as hexane, heptane, dichloromethane, and tetrahydrofuran. Several groups have shown that this alkeneterminated polyisobutylene (PIB) can be modified and used as a polymer support for precious catalysts such as Hoveyda-Grubbs catalysts, ${ }^{1}$ polyoxometalates, ${ }^{2}$ photoredox catalysts, ${ }^{3,4}$ and crosscoupling catalysts. ${ }^{5}$ This highly soluble support makes otherwise insoluble catalysts soluble, ${ }^{6}$ and allows catalytic reactions using PIB supported catalysts to be conducted under homogeneous conditions. These supports also facilitate the isolation and recovery of catalysts by simple phase separation in liquid/liquid biphasic separations after a reaction using heptane and a polar solvent since it is heptane soluble while most polar products are not heptane soluble. Here we describe our initial studies using PIB ligands as polymer supports for recyclable reagents using $\mathrm{PIB}$ as a support for two common reagents used for oxidation of alcohols. These two oxidizing agents were tetra- $n$ propylammonium perruthentate (TPAP) as a catalyst with $N$ methylmorpholine- $N$-oxide as the penultimate oxidant and a stoichiometric oxidizing agent, the ester of 2-iodobenzoic acid (IBX).

A variety of heterogeneous oxidizing agents including catalytic oxidants and stoichiometric have been studied in the past as a solution to the problems of using toxic or expensive conventional oxidants. ${ }^{7}$ For example, while TPAP can be used catalytically as a mild agent to oxidize alcohols to aldehydes or ketones, ${ }^{8} \mathrm{Ru}$ is an expensive metal. While $\mathrm{Ru}$ in these cases is used catalytically by incorporating another oxidant like $\mathrm{N}$ - methylmorpholine- $N$-oxide or molecular oxygen as the penultimate oxidant, there is value in attempting to recycle this transition metal oxidant. ${ }^{7}$ Prior reports have described using both crosslinked polystyrene resins as polymer supports and inorganic or inorganic-hybrid supports to recover and recycle this $\mathrm{Ru}$ oxidant using either NMO or oxygen as the penultimate oxidant. $^{9-11}$

Hypervalent iodine agents too are established oxidants with broad utility in synthetic organic chemistry. ${ }^{12}$ There are many examples of such hypervalent iodine oxidizing agents. One of the most common versions of this reagent is 2-iodoxybenzoic acid (IBX) or derivatives like the ester in Figure 1. IBX is a highly efficient and mild oxidant for oxidation of primary or

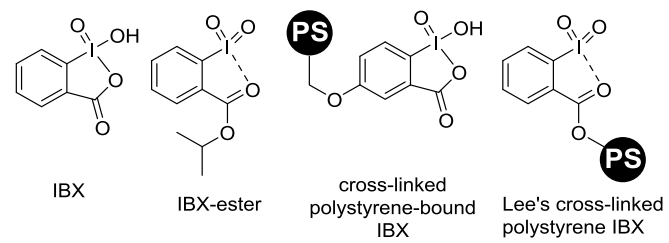

Figure 1. Analogs of IBX including polymer supported analogs.

secondary alcohols. Although IBX has valuable properties, its application in organic synthesis can be limited due to its insolubility. For example, while IBX is soluble in polar aprotic solvents like DMSO, it is relatively insoluble in many common organic solvents. Structural modifications of IBX have also been described to address this solubility issue. For example, Zhdankin and his co-workers described IBX esters and IBX amides that are both stable and soluble in polar organic solvents. ${ }^{13}$ 
Another issue with IBX is that this reagent is most often used stoichiometrically. Thus, recycling of this reagent in an efficient and simple manner would be desirable. Prior studies have emphasized the use of a variety of insoluble supports for this purpose. ${ }^{14}$ These previous studies have reported the use of both inorganic supports like silica gel ${ }^{15}$ and organic polymer supports like cross-linked polystyrene. ${ }^{16,17}$ These studies that immobilized IBX oxidants were precedented by earlier work by the Moss group who also immobilized iodosobenzoate reagents on polystyrene. These reagents are catalysts for phosphate ester hydrolyses and oxidize readily oxidizable substrates. ${ }^{18}$ These insoluble polymer supported oxidants facilitate workup and separation of spent oxidant and product. In some cases recycling of the spent oxidant is also possible. Soluble polymer supported IBX oxidants too have been reported. The Janda group also described the synthesis of soluble polystyrene supported IBX analogs that were shown to be more efficient at oxidizing alcohols than an analogous macroporous insoluble polystyrene supported IBX. ${ }^{19}$ While these soluble oxidants had some advantages and while they too could be separated from products, the separation process involves solvent precipitation and requires excess solvent.

The work here explores a different approach to immobilizing and recycling TPAP and IBX oxidizing agents that uses soluble polymers. It relies on the phase selective solubility of PIB phase anchors after an oxidation process to separate either of these oxidizing agents from products using a liquid/liquid biphasic separation. As we show below, it is straightforward to synthesize both perruthenate oxidants and IBX ester oxidizing agents. We further show that these soluble polymer bound perruthenate and IBX ester oxidants are competent for alcohol oxidation and that they can be separated easily from products. Although recycling the PIB bound perruthenate catalyst was not completely successful, we were able to separate spent PIB-bound IBX esters from products efficiently and were also able to show that it is possible to both readily regenerate and reuse this spent oxidant.

\section{Results and Discussion}

Past work by the Bergbreiter group has shown that commercially available alkene-terminated polyisobutylene oligomers with $M_{\mathrm{n}}$ values from $1000-2300 \mathrm{Da}$ can be converted into a variety of ligands or catalysts with applications in organic synthesis. $^{20}$ The solubility that PIB groups confer on their reactive terminal functionality allows these PIB bound species to both be nonpolar phase anchored species in separations after reactions and highly soluble catalysts for reactions with substrates that can be readily conducted under homogeneous conditions. Inspired by these prior examples, we have explored the potential for these same soluble hydrocarbon oligomer supports as solubilizing and recycling handles for two common oxidizing agents - catalytic tetraalkylammonium perruthenate oxidants and stoichiometric hypervalent iodine oxidants.

We initially examined PIB-bound perruthenate complexes as potential recyclable catalytic oxidants. Using a series of established transformations, alkene-terminated PIB was converted into a heptane soluble PIB oligomer with a terminal tetraalkylammonium iodide functional group. ${ }^{2}$ Then an ion exchange reaction of this PIB-bound ammonium iodide with tetra- $n$-propylammonium perruthenate (TPAP) was used to form $\mathrm{N}, \mathrm{N}$-diethyl, $\mathrm{N}$-methyl, $\mathrm{N}$-polyisobutylammonium perruthenate $\mathbf{2}$ as shown in Figure 2. This ion-exchange reaction was carried out in dichloromethane (DCM) as a solvent. We presumed a 1:1 equilibrium mixture of $\mathbf{1}$ and $\mathbf{2}$ formed when the reaction was carried out with an equal amount of TPAP and 1. The PIBbound perruthenate $\mathbf{2}$ so formed was expected to be phase selectively soluble in heptane versus a polar solvent. This premise was easily tested visually because $\mathbf{2}$ is highly colored. As shown in Figure 3, 2 is indeed phase selectively soluble in heptane versus acetonitrile. This solubility contrasted with that of TPAP which is phase selectively soluble in acetonitrile under the same conditions.

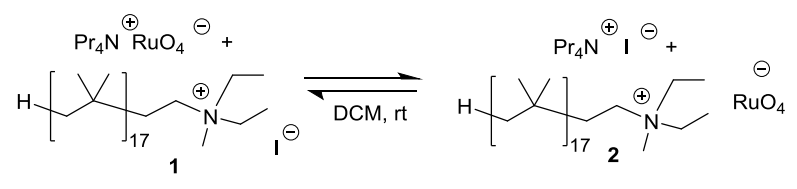

Figure 2. Synthesis of a PIB-bound tetraalkylammonium perruthenate oxidation catalyst.

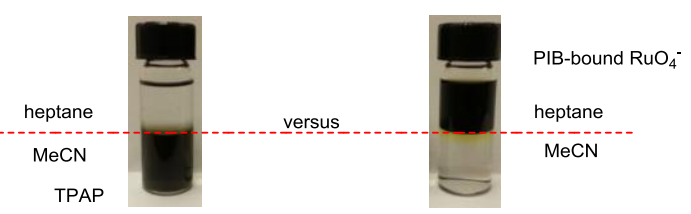

Figure 3. Phase solubility of TPAP and PIB-bound perruthenate

To probe the utility of this heptane soluble TPAP analog as an oxidizing agent, we explored oxidation of 1-phenylethanol to form acetophenone. The PIB-bound perruthenate $\mathbf{2}$ was found to be competent as an oxidation catalyst using NMO as the penultimate oxidant as shown in Table 1. At room temperature in DCM in the presence of molecular sieves, the reaction proceeded to $91 \%$ conversion based on ${ }^{1} \mathrm{H}$ NMR spectroscopy in $12 \mathrm{~h}$. Oxidation of 1-phenylethanol was also successful in heptane though it required elevated temperature $\left(80^{\circ} \mathrm{C}\right)$ to effect a similar $93 \%$ conversion of alcohol to ketone. In the first case, the DCM solvent was removed under reduced pressure. The residue from that reaction was then dissolved in heptane and that solution was extracted with acetonitrile to form an acetonitrile solution of the product ketone and a heptane solution of 2 . In the case of the reaction carried out in heptane, the product was simply extracted with acetonitrile from a heptane solution of 2 . Recycling was then explored. However, attempts to recycle the recovered heptane solution of $\mathbf{2}$ were only modestly successful. A second cycle using the recovered 2 led to only ca. 70\% conversion of 1phenylethanol to acetophenone. Similar problems of diminished yields in recycling of polystyrene supported TPAP had been noted previously. ${ }^{9,21}$ In our experiments, we also noted formation of traces of an insoluble material at the interface of the heptane/acetonitrile phases in the biphasic product separation after an oxidation, leading us to believe that some decomposition of 2 occurred.

Table 1

Catalytic activity of PIB-bound tetraalkylammonium perruthenate in the oxidation of 1-phenylethanol to form acetophenone.

Solvent $\quad$ Time(h) $\quad$ Temperature $\left({ }^{\circ} \mathrm{C}\right) \quad$ Conversion $^{\mathrm{a}}$

\begin{tabular}{|c|c|c|c|}
\hline \multirow[t]{2}{*}{$\overline{\mathrm{DCM}}$} & $12 \mathrm{~h}$ & $25^{\circ} \mathrm{C}$ & Cycle 1: $91 \%$ \\
\hline & & & Cycle 2: $69 \%$ \\
\hline \multirow[t]{2}{*}{ Heptane } & $12 \mathrm{~h}$ & $80^{\circ} \mathrm{C}$ & Cycle 1: $93 \%$ \\
\hline & & & Cycle 2: $73 \%$ \\
\hline
\end{tabular}

${ }^{a}$ The conversion of substrate to product was measured using ${ }^{1} \mathrm{H}$ NMR spectroscopy.

Decomposition of the PIB ammonium salt that occurred by a base-induced elimination reaction involving the ammonium salt could explain decomposition of $\boldsymbol{2}$. We briefly explored an alternative a tetraalkylammonium salt binding group by preparing a PIB-bound $\mathrm{N}$-methylimidazolium salt as shown in Figure 4. In this synthesis, $\mathrm{NaH}$ was used to promote reaction of the imidazole with the PIB-bound primary alkyl bromide $\mathbf{3}$ to 
form the PIB-bound imidazole 4. This PIB-bound imidazole 4 was then allowed to react with iodomethane to form a PIB-bound $\mathrm{N}$-methylimidazolium iodide $\mathbf{5}$. Finally using the same procedure above (Figure 2), this PIB-bound $N$-methylimidazolium salt 5 was converted to form the tetraalkylammonium perruthenate $\mathbf{6}$ by an ion-exchange reaction.

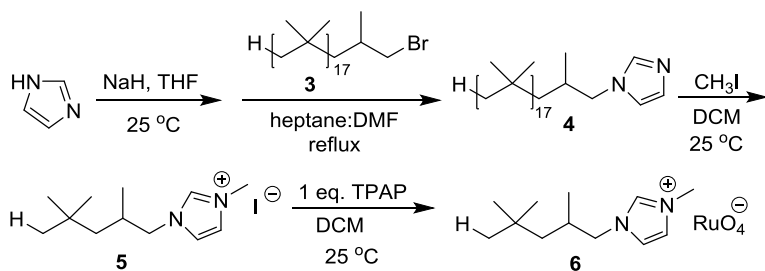

Figure 4. Synthesis of a $N$-methylimidazolium perruthenate.

To explore the utility and recyclability of $\mathbf{6}$ as a catalytic oxidant, $10 \mathrm{~mol}$. \% of 6 was used to oxidize benzyl alcohol using NMO as the penultimate oxidant. The results are shown in Table 2. However, while this PIB-bound $N$-methylimida-zolium perruthenate catalyst too worked well in cycle 1, it like 2 had activity that decreased to ca. $63 \%$ in cycle 2 in either heptane or DCM.

Table 2.

Catalytic activity of PIB-bound imidazolium perruthenate 6 in oxidation of benzyl alcohol to form benzaldehyde.

$\begin{array}{llll}\text { Solvent } & \text { Time }(\mathrm{h}) & \text { Temperature }\left({ }^{\circ} \mathrm{C}\right) & \text { Conversion }^{\mathrm{a}}\end{array}$

\begin{tabular}{|c|c|c|c|}
\hline DCM & $3 \mathrm{~h}$ & $25^{\circ} \mathrm{C}, 3 \mathrm{~h}$ & Cycle 1: $98 \%$ \\
\hline
\end{tabular}

Heptane $\quad 12 \mathrm{~h} \quad 80^{\circ} \mathrm{C}, 12 \mathrm{~h} \quad$ Cycle $1: 86 \%$

Cycle 2: $61 \%$

${ }^{a}$ The conversion of substrate to product was measured using ${ }^{1} \mathrm{H}$ NMR spectroscopy.

Since the PIB-bound perruthenate complexes 2 and $\mathbf{6}$ like those described by Ley ${ }^{9,21}$ gave lower conversions in cycle 2, we proceeded to examine hypervalent iodine oxidants that have been immobilized by others on insoluble supports and successfully recycled. The synthesis of these PIB-bound IBX analogs was readily accomplished as shown in Figure 4. Alkene-terminated PIB oligomers were subjected to a hydroboration-oxidation reaction to generate the PIB supported alcohol $\mathbf{8}$ which was esterified to form the PIB supported ester 9 using 2-iodobenzoic acid with DCC and DMAP. After purification by column chromatography, the PIB supported ester 9 was oxidized using tetrabutylammonium oxone (TBA-Ox) and $\mathrm{MeSO}_{3} \mathrm{H}$ in DCM to form the desired PIB supported IBX ester 10. In this chemistry, the oxidant, TBA-Ox, was prepared by a procedure described by Borhan $^{22}$ and its oxidative activity $(82 \%)$ was measured by iodometric titration before its use in formation of $\mathbf{1 0}$. The conversion of PIB-supported ester to PIB supported IBX ester was determined by using ${ }^{1} \mathrm{H}$ NMR spectroscopy looking at the ratio of the diastereotopic $-\mathrm{CH}_{2} \mathrm{O}$ - signal of $\mathbf{9}$ at $4.33 \mathrm{ppm}$ and the signal for the same group in $\mathbf{1 0}$ at $4.03 \mathrm{ppm}$. The average conversion of $\mathbf{9}$ to $\mathbf{1 0}$ in six different reactions was $87 \%$ and ranged from $81 \%$ to $94 \%$. The solution of the PIB supported IBX

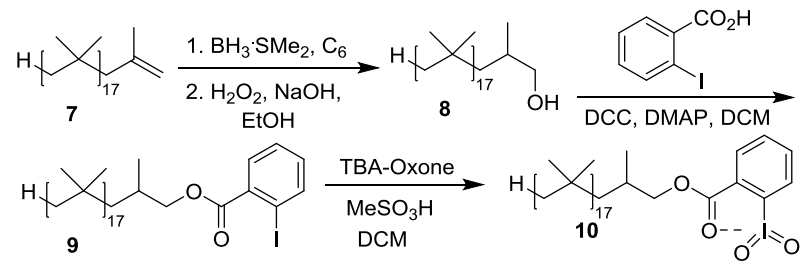

Figure 5. Synthesis of PIB supported IBX ester. ester so formed was then directly used in oxidation reactions without isolation of $\mathbf{1 0}$.

We first looked at the oxidation of benzyl alcohol to benzaldehyde to determine the effectiveness of $\mathbf{1 0}$ as an oxidizing agent and to determine the best reaction conditions (Table 3) measuring the extent of oxidation of benzyl alcohol to benzaldehye by ${ }^{1} \mathrm{H}-\mathrm{NMR}$ spectroscopy. These studies showed that the oxidation reaction did not proceed well unless acid promoters such as trifluoroacetic acid (TFA) or boron trifluoride etherate were added. The efficiency of acid promoters like boron trifluoride etherate has also been noted in oxidations with the low molecular weight IBX. ${ }^{13}$ Interestingly, we also observed an unexpected solvent effect in that the oxidation of benzyl alcohol worked better in heptane than in DCM, the typical solvent used for IBX oxidants. While both promoters facilitated the conversion of benzyl alcohol to benzaldehyde, TFA was judged less desirable for two reasons. First, with $\mathbf{1 0}$ as the oxidizing agent using TFA as a promoter, the reaction time with TFA was significantly longer than the time required for this same reaction with a crosslinked polystyrene supported IBX ester. Second, when we used TFA we also observed the formation of a small amount of a white precipitate during the reaction. We could not identify what this precipitate was as it was insoluble in $\mathrm{CDCl}_{3}$, $d_{6}$-DMSO, and $d$-benzene. Since this problem was not seen with oxidations that used boron trifluoride etherate as a promoter and since the reactions with boron trifluoride ethereate led to near quantitative conversions of benzyl alcohol to benzaldehyde in less than $30 \mathrm{~min}$, we focused our attention on reactions with this acid promoter.

Table 3.

Conversions in oxidation of benzyl alcohol with 10 at $25^{\circ} \mathrm{C}$ $\mathbf{1 0}$ (equiv) Solvent $\quad$ Promoter $\quad$ Time (h) Conversion $^{\mathrm{a}}$

\begin{tabular}{lllll}
\hline 2.38 & DCM & none & 24 & $4 \%$ \\
2.26 & heptane & none & 24 & $33 \%$ \\
1.76 & DCM & 1 equiv TFA & 24 & $30 \%$ \\
2.42 & heptane & 1 equiv TFA & 24 & $94 \%$ \\
1.67 & heptane & none & 1 & $100 \%$ \\
1 & heptane & 1.0 equiv & $<0.5$ & $97 \%$ \\
& & $\mathrm{BF}_{3} \mathrm{OEt}_{2}$ & & \\
1.64 & $\mathrm{CDCl}_{3}$ & 1.4 equiv $^{\mathrm{b}}$ & 0.2 & $91 \%$ \\
& & $\mathrm{BF}_{3} \mathrm{OEt}_{2}$ & & \\
\hline
\end{tabular}

a The conversion of substrate to product was measured using ${ }^{1} \mathrm{H}$ NMR spectroscopy.

${ }^{\text {b }}$ This reaction was carried out at $80^{\circ} \mathrm{C}$.

Given success with benzyl alcohol oxidation, the scope of this PIB supported IBX ester $\mathbf{1 0}$ as an oxidation reagent with $\mathrm{BF}_{3} \cdot \mathrm{OEt}_{2}$ as promoter and heptane as the solvent was tested using a collection of other substrates. Table 4 shows that the PIBbound oxidizing agent $\mathbf{1 0}$ efficiently oxidizes a variety of different types of alcohols with conversions ranging from $95 \%$ $100 \%$.

While the PIB-IBX ester oxidant $\mathbf{1 0}$ is effective as a stoichiometric reagent, our original goal was to recycle oxidation catalysts or reagents. While this did not work as well as hoped with $\mathbf{2}$ or $\mathbf{6}$, recycling spent $\mathbf{1 0}$ was successful. A key advantage 
was that unreacted or spent $\mathbf{1 0}$ could be easily separated from products since PIB is a phase selectively heptane soluble phase anchor. This coupled with the fact that product ketones and aldehydes produced in the oxidation reaction are likely more soluble in acetonitrile than heptane suggested that spent $\mathbf{1 0}$ could be separated from products and reoxidized. That proved to be correct.

Experiments that reused a heptane solution of benzyl alcohol as a substrate for an oxidation using $\mathbf{1 0}$ used 2 equivalent of $\mathbf{1 0}$ as an oxidant and separated the benzaldehyde product from spent $\mathbf{1 0}$ using an acetonitrile extractions, relying on the phase selective solubility of the benzaldehyde product in acetonitrile and the

Table 4.

Survey of substrate scope of oxidation reactions using $\mathbf{1 0}$

Substrates

a The conversion of substrate to product was measured using ${ }^{1} \mathrm{H}$ NMR spectroscopy.

phase selective solubility of unreacted and spent $\mathbf{1 0}$ in heptane. These recycling experiments were carried out with both benzyl alcohol and 1-phenyl-2-propanol. The PIB-IBX ester $\mathbf{1 0}$ was found to be recyclable up to 5 times as shown in Table 5. After a biphasic separation, the heptane solution of the spent PIB oxidant was not generally isolated but was simply reoxidized using TBAOx as described in Figure 5. However, if desired, the spent oxidant could be isolated and analyzed by ${ }^{1} \mathrm{H}$ NMR spectroscopy. In cases where that analysis was carried out, the PIB bound aryl ester present after the oxidation was a mixture of $\mathbf{9}$, unreacted $\mathbf{1 0}$, and the PIB ester of the iodosyl aryl iodide which was not active for alcohol oxidation under our conditions.

Table 5

Conversions for alcohol oxidations in five recycles of the PIB-bound IBX ester 10.

\begin{tabular}{|c|c|c|c|c|c|}
\hline \multirow[b]{2}{*}{$\mathrm{R}-\mathrm{OH}$} & \multicolumn{2}{|c|}{2 equiv. PIB-IBX ester 10} & & & 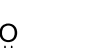 \\
\hline & \multicolumn{2}{|c|}{$\begin{array}{c}\mathrm{BF}_{3} \mathrm{OEt}_{2} \text {, heptane } \\
20 \mathrm{~min}, 25^{\circ} \mathrm{C}\end{array}$} & & & \\
\hline Substrates & Cycle 1 & Cycle 2 & Cycle 3 & Cycle 4 & Cycle 5 \\
\hline & $>94 \%$ & $>99 \%$ & $>99 \%$ & $>99 \%$ & $80 \%$ \\
\hline & $>99 \%$ & $>98 \%$ & $100 \%$ & $>92 \%$ & $100 \%$ \\
\hline
\end{tabular}

a The conversion of substrate to product was measured using ${ }^{1} \mathrm{H}$ NMR spectroscopy.

\section{Conclusions}

The results above show that PIB-bound versions of TPAP and IBX are both competent oxidants for oxidations of alcohols to ketones and aldehydes. However, while TPAP can be used catalytically with NMO as a penultimate oxidant, recycling of a perruthenate catalyst ionically immobilized on PIB was only modestly successful. TPAP-catalyzed oxidations were also slower in heptane solvents. Oxidations with a recyclable PIBbound IBX were more successful. In the case of PIB-IBX, the PIB-bound hypervalent iodine oxidant was highly soluble in a solvents like heptane, toluene, methylene chloride, THF, and chloroform solvents. It was competent for oxidations of a variety of primary and secondary alcohols in the presence of $\mathrm{BF}_{3^{-}}$ etherate as a Lewis acid promoter and the reaction was unexpectedly faster in heptane than in DCM. The spent oxidant also could be reused after an oxidation for as many as five times. This suggests that PIB may be generally useful in recycling other stoichiometric reagents. The observation that PIB-IBX was more reactive in heptane than in DCM is in our opinion also interesting as it suggests that reactions in heptane or other saturated hydrocarbon solvents might merit more attention especially if a readily available alkane soluble PIB phase anchor can be designed to make reagents and/or substrates usefully heptane soluble.

\section{Acknowledgments}

We would like to thank the National Science Foundation (Grant CHE-1362735) and the Robert A. Welch Foundation (Grant A-0639) for supporting this research as well as the Department of Chemistry of Texas A\&M University for their financial support.

\section{References and notes}

1. Suriboot, J.; Hobbs, C. E.; Guzman, W.; Bazzi, H. S.; Bergbreiter D. E. Macromolecules 2015, 48, 5511-5516.

2. Yahya, R.; Craven, M.; Kozhevnikov, E.; Steiner, A.; Samunual, P.; Kozhevnikov, I.; Bergbreiter, D. E. Catal. Sci. Technol. 2015 5, 818-821.

3. Liang, Y.; Bergbreiter, D. E.; Catal. Sci. Technol. 2016, 6, 215 221.

4. Rackl, D.; Kreitmeier, P.; Reiser, O. Green Chem. 2016, 8, 214.

5. Khamatnurova, T.V.; Zhang, D.; Suriboot, J.; Bazzi, H.S.; Bergbreiter, D. E. Catal. Sci. Technol. 2015, 5, 2378-238.

6. Chao, C.-G.; Bergbreiter, D. E. Catal. Commun. 2016, 77, 89-93.

7. Ley, S. B.; Norman, J.; Griffith, W. P.; Marsden, S. P. Synthesis 1994, 639-666.

8. Ciriminna, R.; Pandarus, V.; Beland, F.; Xu, Y.-J.; Pagliaro, M. Org Proc. Res. Develop. 2015, 19, 1554-1558.

9. Hinzen, B.; Ley, S.V. J. Chem. Soc., Perkin Trans. 1 1997, $1907-$ 1908.

10. Pagliaro, M.; Ciriminna, R. Tetrahedron Lett. 2001, 42, 45114514.

11. Matsumoto, T.; Ueno, M.; Wang, N.; Kobayashi, S. Chem. -An Asian J. 2008, 3, 239-243

12. Yoshimura, A.; Zhdankin, V.V. Chem. Rev. 2016, 116, 3328 3435 .

13. Zhdankin, V.V.; Koposov, A. Y.; Litvinov, D. N.; Ferguson, M. J.; McDonald, R.; Luu, T.; Tykwinski, R. R. J. Org. Chem. 2005 , 70, 6484-6491

14. Yusubov, M. S.; Zhdankin, V. V. Mendeleev Commun. 2010, 20 185-191

15. Mülbaier, M.; Giannis, A. Angew. Chem. Int. Ed. 2001, 40, $4393-$ 4394.

16. Sorg, S.; Mengel, A.; Jung, G.; Rademann, J. Angew. Chem. Int Ed. 2001, 40, 4395-4397.

17. Chung, W.; Kim, D.; Lee, Y. Tetrahedron Lett. 2003, 44, 92519254.

18. Moss, R. A.; Bolikal, D.; Durst, D.; Hovanec J. W. Tetrahedron Lett. 1988, 29, 2433-2436.

19. Reed, N. N.; Delgado, M.; Hereford, K.; Clapham, B.; Janda, K D. Bioorg. Med. Chem. Lett. 2002, 12, 2047-2049.

20. Li, J.; Sung, S.; Tian, J.; Bergbreiter, D.E. Tetrahedron 2005, 61, 12081-12092.

21. Ley, S. V.; Ramarao, C.; Smith, M. D. Chem. Commun. 2001, 2278-2279.

22. Travis, B. R.; Ciaramitaro, B. P.; Borhan, B. Eur. J. Org. Chem. 2002, 3429-3434. 


\section{TYPICAL OXIDATION REACTION}

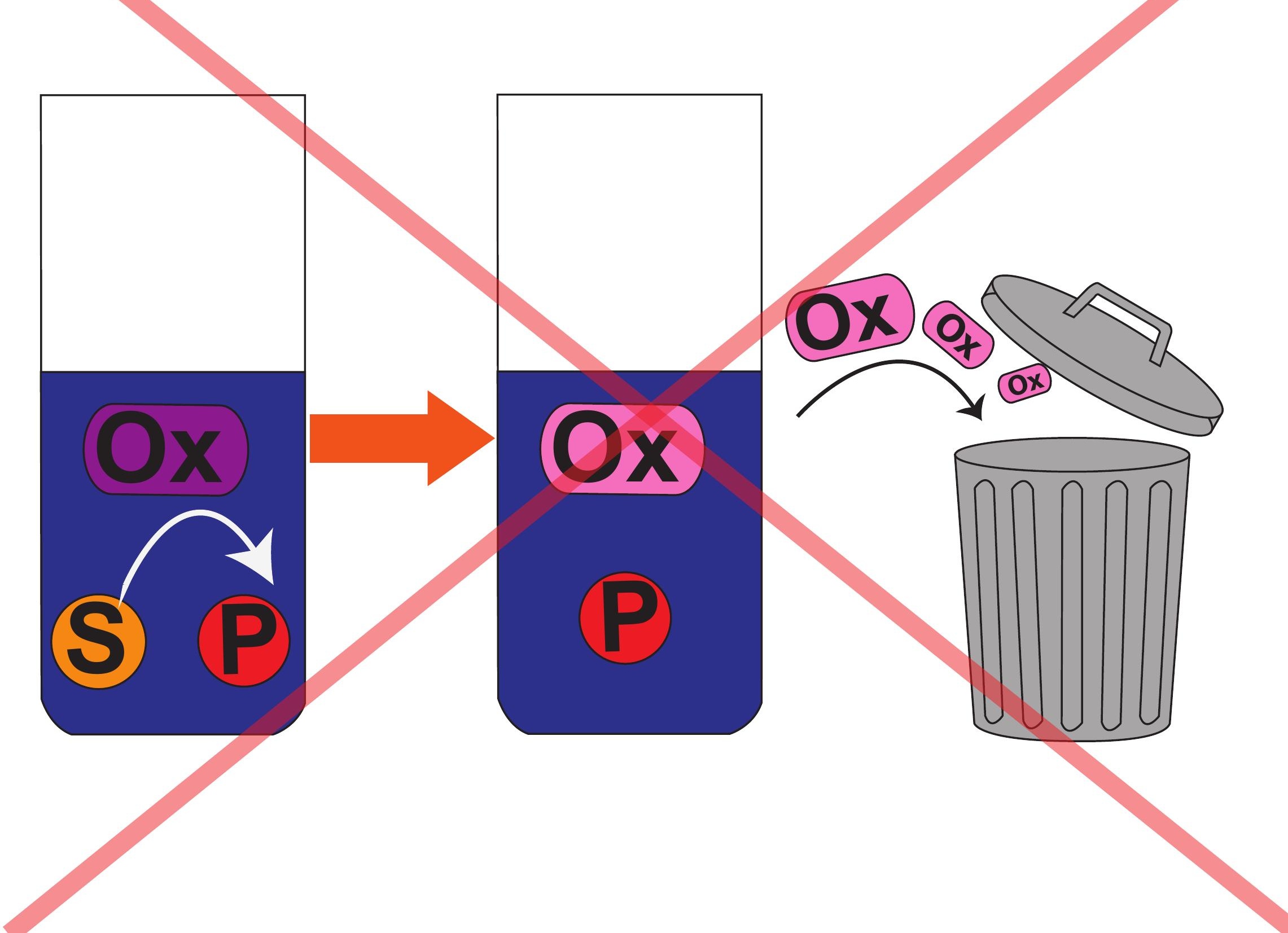

RECYCLABLE PIB-BOUND OXIDIZING AGENTS

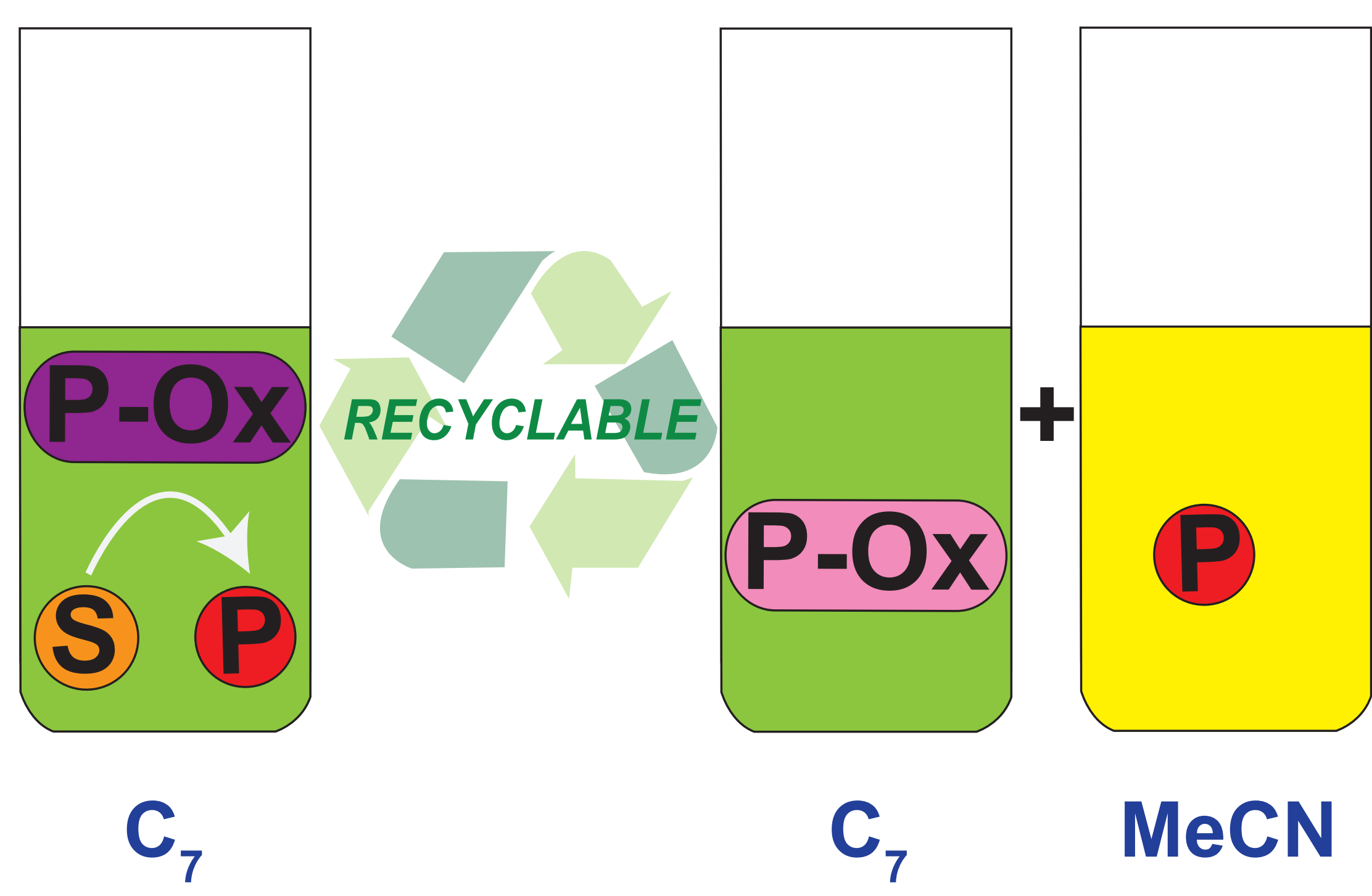

